\title{
Single nucleotide polymorphisms in the cyp2C8 and nat2 genes and treatment outcomes in patients suffering from uncomplicated malaria in Garoua, Northern Region of Cameroon
}

\begin{abstract}
The human Cytochrome (cyp2C8) and $\mathrm{N}$-acetyltransferase II (nat2) genes may vary between individuals resulting in differences in anti-plasmodial treatment responses. We set out in this study to determine the association between cyp2C8 and nat 2 gene phenotypes, with treatment failures among children with uncomplicated malaria in Cameroon. Treatment outcomes were assessed after, children $(n=235)$ were deparasitized with artemisinin based combination therapies (ACTs) (Artesunate-Amodiaquine or Sulfadoxine-PyrimethamineAmodiaquine) and followed up for 28 days. Dried blood spots were used for nested PCR followed by restriction enzyme analysis with KpnI, TaqI, and BamHI for the detection of polymorphisms in cyp2C8 and nat 2 genes. Two alleles in the study population were identified with the predominance of the wild-type cyp2C8*1 allele (76\%) and the fast metabolisers phenotype (70\%). The nat $2 * 6$ genotype was highest at $39 \%$ and nat $2 * 5 / 6$ highest at $28.12 \%$. Slow metabolizers were found at a frequency of $61.4 \%$ and intermediate metabolizers at $28.2 \%$. Prior to PCR corrections $63.5 \%$ patients on ACTs had an Adequate Clinical and Parasitological Response (ACPR), while 24\% experienced treatment failure (ETF), and $12.5 \%$ Late Parasitological Failure (LPF). Individuals with ETF (Odd's Ratio $=4.792$ and $p$-value $=0.020$ ) clustered with the fast acetylation phenotype compared to slow and intermediate metaboliser phenotypes. The distance-based clustering analysis using the Jaccard index for calculation of genetic distances showed patients who are intermediate metabolizers and carried wild-type Pfdhfr-S108 and Pfdhps-C58 formed one cluster. Slow metabolizers carrying the 2 resistance markers $108 \mathrm{~N}$ and $58 \mathrm{R}$ formed another cluster, while fast metabolizers with wild-type genotype at drug resistance codons clustered together. We conclude that the cyp $2 \mathrm{C} 8$ wild-type allele and the fast metabolizers predominate in the north of Cameroon. Likewise, the nat $2 * 5 / 6$ and its slow metabolizer status. ACPR to antimalarial treatment may be well associated with a nat2 slow metabolizer phenotype.
\end{abstract}

Keywords: malaria, cyp $2 C 8$, nat2, metabolysers, treatment failure, adequate clinical and parasitological response
Volume 7 Issue 4 - 2019

Rose Ngono Mballa,' Jean Paul Chedjou, ${ }^{2}$ Randolph Ngwafor, ${ }^{3}$ EkolloAristid, ${ }^{2}$ Innocent Alii, ${ }^{4,5}$ AkindehNji, ${ }^{2,4}$ Forsah-Achu Dorothy, ${ }^{3}$ Ekoyol Ekobe Germaine, ${ }^{3}$ Olivia Achondu, ${ }^{2}$ Dinga-Nyoh Mbeku Veh, ${ }^{6}$ Wilfred Fon Mbacham 4,5

'Department of Pharmacology and Traditional Medicine, Faculty of Medicine and Biomedical Sciences, University of Yaounde I, Cameroon

${ }^{2}$ Department of Biochemistry, University of Yaounde I, Yaounde, Cameroon

${ }^{3}$ Ministry of Health, Cameroon National Malaria Control Program 4MARCAD-DELTAS Program, University of Yaounde I, Cameroon Department of Biochemistry, University of Dschang, Cameroon ${ }^{6}$ Department of Biochemistry, Faculty of Medicine and Biomedical Sciences, University of Yaounde I, Cameroon

Correspondence: : Ngono Mballa Rose, Department of Pharmacology and Traditional Medicine, Faculty of Medicine and Biomedical Sciences, University of Yaounde I, Cameroon, Tel +237 67763548I,Email princessrose@hotmail.fr

Mbacham Fon Wilfred, Department of Biochemistry, University of Dschang, Dschang, Cameroon, Tel +237 677579180, Emailwfmbacham@yahoo.com

Received: June II, 2019 | Published: July 02, 2019

\section{Introduction}

Malaria is an infectious disease transmitted by mosquitoes in humans and other animals caused by the parasitic protozoan, Plasmodium. According to the latest statistics of the World Health Organization, ${ }^{1} 216$ million cases of malaria occurred worldwide in 2016, an increase of about 5 million cases compared to 2015 . Most cases in 2016 occurred in the WHO regions of Africa $(99.7 \%$ P. falciparum), South-East Asia (7\%) and Eastern Mediterranean (2\%). The WHO Cameroon epidemiological bulletin reported an increase of 70,420 new cases $(10,52 \%)$ in 2016. Malaria remains a public health problem in Cameroon with enormous morbidity and mortality, partly caused by resistance to conventional antimalarial drugs. $^{2}$ But the unprecedented malaria epidemic of October 2013 in Maroua (Far North Region of Cameroon) which left more than 2,000 people hospitalized and at least 600 deaths, has called into question the efficacy of antimalarial drugs in this northern region. The implementation of Seasonal Malaria Chemoprevention (SMC) in subSaharan Africa in March 2012, was reported by WHO, after trials of Sulfadoxine-Pyrimethamine (SP) in combination with Amodiaquine
(SPAQ) in children below 5 years. The trial demonstrated safety and high efficacy in Mali, Central African Republic and Cameroon. ${ }^{3-6}$ SPAQ given at monthly intervals has been shown to be $75 \%$ protective against uncomplicated and severe malaria in children under five years. ${ }^{7}$

The Ministry of Health decided with the support of technical partners to implement WHO's Seasonal Malaria Chemoprevention (SMC) program; ${ }^{7,8}$ which recommends that SMC with SPAQ is administered in areas with highly seasonal malaria transmission specifically in the Sahel sub-region of sub-Saharan Africa. $P$. falciparum is still sensitive to both these antimalarial drugs. As part of the implementation of the SMC strategy in Cameroon, a clinical trial was necessary to assess the efficacy of SPAQ versus ASAQ as the comparator drug in the Northern Regions of Cameroon. The age group in this trial was extended from 6 months to 10 years. To this end, two main reactions that take place in the liver are essential; Phase I and Phase II reactions. While Phase I reaction is primarily catalyzed by Cytochrome P450 enzymes (CYP), Phase II reactions are catalyzed by many enzymes including Acetyltransferase (NAT2), Glucuronosyl-transferase, Glutathione-s-transferase and Sulpho- 
transferases. ${ }^{9}$ However, the effective metabolization pathway of certain drugs show they/these drugs are metabolized by a single enzyme (metoprolol by CYP2D6), whereas other drugs involve two or more enzymes (warfarin by CYP1A2, CYP2D6 and CYP3A4). ${ }^{10}$ The polymorphisms in cyp $2 \mathrm{C} 8$ and nat2 genes may influence antimalarial treatment outcomes among individuals suffering from uncomplicated malaria. To better understand such differences in the Cameroon setting, we characterized the single nucleotide polymorphisms (SNPs) of cyp $2 C 8$, nat 2 (nat $2 * 5$, nat $2 * 6$ and nat $2 * 7$ ) and their relationship with the treatment response of the population of the Northern region.

\section{Materials and methods}

\section{Study area}

The study was conducted in Garoua, which is situated in the north of Cameroon $\left(06^{\circ} 24^{\prime} \mathrm{N}, 10^{\circ} 46^{\prime}\right)$. Garoua serves as a river port in years when the rainfall is abundant. Situated in the river Benue basin, it receives an average annual rainfall of $380 \mathrm{~mm}$. It has about 4 months of rainy season. The mean temperatures for most of the year are about $31^{\circ} \mathrm{C}$ and the vegetation is guinea-savannah. The population is predominantly of the Fula ethnic group and comprises of cattle grazers. A few have taken to trading in small provision merchandise with neighbouring Nigeria.

\section{Screening and enrolment}

Eligible children aged 6 to 120 months with acute uncomplicated falciparum malaria were screened at the outpatient department and informed consent obtained from parents or guardians. Patients meeting the inclusion criteria were randomized to trial allocation and pre-treatment investigations (clinical and laboratory assessments) conducted. Criteria for inclusion were; children of either gender, suffering from acute uncomplicated $P$. falciparum malaria confirmed by microscopy, or presenting with fever (axillary temperature $\geq$ $37.5^{\circ} \mathrm{C}$ ) or having a history of fever in the preceding $24 \mathrm{~h}$; ability to ingest tablets orally; willingness and ability to attend the clinic on stipulated follow-up days. A presentation was made of the anticipated risks and benefits, the discomfort to which the subjects were exposed, as well as the right to interrupt the participation at any time on their own free-will. A total of 235 patients were enrolled for the study.

\section{ACT Administration and follow-up}

Children $(\mathrm{n}=235)$ were randomized to receive ArtesunateAmodiaquine and Sulfadoxine-Pyrimethamine-Amodiaquine in the ratio of 2:2 respectively. The first dose of the anti-malaria's was administered in the hospital and the rest of the tablets were administered at home, according to the prescription. The medication was administered with water after a meal. Follow up of children was ensured by community health workers (CHW) who visited participants daily in the first three days and reported any complaints from the mother. They also visited these mothers with complaints on other days besides the protocol stipulated days (D7, D14, D28, D35 and D42 after first dose). Complaints included but were not limited to any signs and symptoms suggestive of malaria or toxicity, date of onset, duration of symptoms, severity of malaria or other treatment administered and patient outcomes. Participants were followed up for 42 days post ACT-administration.

\section{Sample collection and DNA extraction}

Finger-prick blood was collected and spotted on filter paper at inclusion and during follow up on days D0 for genomic (or molecular analyses!). Blood spots on the filter paper were excised with a sterile pair of surgical scissors. DNA was extracted from dried blood spots heating at $100^{\circ} \mathrm{C}$ in Chelex-100 in buffered Tris-EDTA as previously described. ${ }^{11}$ The DNA was stored in a Tris-EDTA buffer at $-20^{\circ} \mathrm{C}$ until allelic discrimination analysis was done by PCR-RFLP.

\section{Genotyping single nucleotide polymorphisms in сур2 28 and nat2 gene}

Amplification of the cyp2C8 gene was done according to the adapted approach of Dai et al., ${ }^{12}$ using primers (cyp2C8 F:5'-AAGATACATATATCTTATGACATG-3 \& cyp2C8 R:5'ATCCTTAGTAAATTACAGAA GG-3'). For a total of $25 \mu 1$, the reaction mixture (NEBiolabs) was composed of PCR water, buffer (10Xthermopol buffer), $10 \mathrm{mM}$ dNTPs $(200 \mu \mathrm{M}$ of each deoxyribonucleotide), $0.8 \mu \mathrm{M}$ of each primer, $1.25 \mathrm{U} / \mu \mathrm{l}$ of Taq polymerase DNA and $5 \mu$ of DNA extract. The T3 thermal cycler (Biometra, UK) was used for the PCR amplification of target genes. Cycling conditions were as follows: $94^{\circ} \mathrm{C}$ for 5 minutes (predenaturation), 45 cycles of $94^{\circ} \mathrm{C}$ for 20 secs (denaturation), $55^{\circ} \mathrm{C}$ for 20 secs (annealing), $72^{\circ} \mathrm{C}$ for 20 secs (extension). Final extension of amplicons was carried out at $72^{\circ} \mathrm{C}$, after which PCR products were stored at $4^{\circ} \mathrm{C}$ for immediate use or $-20 / 80^{\circ} \mathrm{C}$ for long-term use. The restriction enzyme $\mathrm{Bcl} \mathrm{I}$ was used in digesting the cyp $2 \mathrm{C} 8$ gene according to a previously described protocol. ${ }^{12}$ Digestion was carried out in a volume of $20 \mu$ containing PCR water (NEBIolabs), buffer (10X buffer, manufacturer), $4 \mathrm{U} / \mu \mathrm{l}$ of Bcl I restriction enzyme and $8 \mu \mathrm{l}$ of cyp $2 \mathrm{C} 8$ amplicon. The reaction mixture was incubated at $50^{\circ} \mathrm{C}$ for $12 \mathrm{H}$.

The most common alleles of nat 2 gene in African populations were genotyped/screened for the presence of the following mutations: C481T (rs 1799929, amino acid change L161L), G590A (rs1799930, amino acid change R197Q), A803G (rs1208, amino acid change K268R) and G857A ( $r s 1799931$, amino acid change G286E). The primers used to amplify the gene were: nat $2(+) 5$ '-GCCTCAGGTGCCTTGCATTT-3' and nat 2 (-) 5'-CGTGAGGGTAGAGAGGATAT-3'. The amplification was carried out using a T3 thermal cycler (Biometra, UK). Each PCR cycle was performed in a total volume of $25 \mu \mathrm{l}$ containing: nuclease free water, 10Xthermopol buffer, $10 \mathrm{mMdNTPs}(200 \mu \mathrm{M}$ of each deoxyribonucleotide), $20 \mathrm{pmol}$ primer and $5 \mathrm{U} / \mu \mathrm{L}$ Taq polymerase and $3 \mathrm{ng}$ of gDNA. After initial denaturation at $95^{\circ} \mathrm{C}$ for $5 \mathrm{~min}, 30$ cycles of amplification were carried out with denaturation at $95^{\circ} \mathrm{C}$ for $50 \mathrm{~s}$, annealing at $55^{\circ} \mathrm{C}$ for $50 \mathrm{~s}$ and extension at $72^{\circ} \mathrm{C}$ for $50 \mathrm{~s}$, followed by a final extension at $72^{\circ} \mathrm{C}$ for $5 \mathrm{~min}$. To confirm the presence of nat 2 alleles, PCR products were electrophoresed on a $2 \%$ agarose gel and polymorphisms determined by restriction endonuclease digestion of amplified gene fragments as described. ${ }^{13}$ The amplicons were digested under conditions stipulated for the restriction enzymes KpnI and BamHI (NEBiolabs, USA) $\left(37^{\circ} \mathrm{C}\right.$ for $16 \mathrm{~h}$ while Taq I digested at $65^{\circ} \mathrm{C}$ for $16 \mathrm{~h}$ ). The digestion reaction was followed by inactivation at $80^{\circ} \mathrm{C}$ for $20 \mathrm{~min}$. Digested and undigested fragments of each sample were electrophoresed on a 2\% agarose gel stained with Ethidium Bromide and the pattern of migration analysed by UV trans-illumination. Different alleles and combinations of alleles of the cyp2C8 and nat 2 genes were determined for each sample according to the migration pattern and information indicated.

\section{Classification of acetylator genotypes}

The genotypic frequency of the cyp2C8 genes were distinguished as fast metabolizers (cyp2C8*1) or slow metabolizers (cyp2C $8 * 2)$. The 
nat 2 acetylator genotypes were established according to previously published data. ${ }^{25}$ Homozygotes (nat $2 * 4 /$ nat $2 * 4$ ) or heterozygotes (nat $2 * 4 /$ nat $2 * 5$, nat $2 * 4 /$ nat $2 * 6$ and nat $2 * 4 /$ nat $2 * 7$ combinations) for the dominant nat $2 * 4$ wildtype allele were classified as fast acetylator genotypes, while homozygotes of the mutant alleles (nat $2 * 5$, nat $2 * 6$ and $n a t 2 * 7)$ were classified as slow acetylator genotypes.

Patients harbouring the cyp $2 C 8^{*} 1$ genotype were phenotypically distinguished/categorized as fast metabolizers, while patients typed with cyp $2 \mathrm{C}^{*} 2$ as slow metabolizers. nat 2 acetylator genotypes were established according to previously published data. ${ }^{14}$ Patients were classified as fast acetylators, if they were homozygous (nat $2 * 4$ ) nat $2 * 4$ ) or heterozygous (a combination of nat $2 * 4 /$ nat $2 * 5$, nat $2 * 4$ / nat $2 * 6$, and nat $2 * 4 /$ nat $2 * 7$ ) to the dominant nat $2 * 4$ wild-type allele. On the converse, they were classified as slow acetylators if they were homozygous with the mutant alleles (nat $2 * 5$, nat $2 * 6$ and nat $2 * 7$ )

\section{Distance-based clustering analysis}

Genetic distances that have served for the establishment of the phylogenetic tree were calculated with the formula below:

$\mathrm{D}_{\mathrm{X} / \mathrm{Y}}=\mathrm{N}_{\mathrm{XY}} /\left(\mathrm{N}_{\mathrm{X}}+\mathrm{N}_{\mathrm{Y}}-\mathrm{N}_{\mathrm{XY}}\right) ; \mathrm{D}_{\mathrm{X} / \mathrm{Y}}$ - the genetic distance between $\mathrm{X}$ and $\mathrm{Y}$,

$\mathrm{N}_{\mathrm{X}}$ - the number of electrophoresis bands of $\mathrm{X}$,

$\mathrm{N}_{\mathrm{Y}}$ - the number of electrophoresis bands of $\mathrm{Y}$,

$\mathrm{X} \& \mathrm{Y}$ represent the different haplotypes basis on nat2 genes, $P f d h f r$ and $P f d h p s$. These distances were used to draw a distance tree using the software MEGA 5.05

\section{Data analysis}

Data was entered using Microsoft Access 2007, CS-Pro and analysed using Statistical Package for the Social Sciences (SPSS). The distance migrated by each gene was measured against that of molecular weight marker and recorded using a Microsoft 2016 Excel spreadsheet. The molecular size of each gene was determined graphically from the curve of $\log _{10}$ of molecular weight marker against the distance of migration.

Efficacy of anti-malaria drugs: PCR-adjusted parasitological cure rates were analysed using Fisher's exact test, odds ratios for likelihood of cure with $95 \%$ confidence intervals, Kaplan-Meier survival analysis.

Safety of anti-malaria drugs: Prevalence of Adverse Events were analysed by the Fisher's exact test. Changes of biological safety tests
\& vital signs: Wilcoxon's Signed Rank test or paired t-test. Statistical significance was set at $\alpha=0.05$ for all tests.

Allelic frequencies for the cyp2C8 and nat 2 gene were obtained using the Hardy-Weinberg equilibrium formula. Phenotypic frequencies were obtained by dividing the total number of a particular phenotype by the total number of patients. The relationship between the cyp $2 \mathrm{C} 8$, nat 2 gene and treatment outcomes were analyzed by the $\chi^{2}$ test. The odds ratio (OR) was evaluated using a confidence interval of $95 \%$, for the susceptibility of early treatment failure for fast acetylators and late parasitological failure for slow acetylators. A p-value less than 0.05 were considered statistically significant.

\section{Ethical clearance}

Ethical approval was obtained for the study from the National Ethics Committee for Health Research on human subjects in Cameroon.

\section{Results}

A total of 235 children were enrolled from North regions. The number of children randomised to the ASAQ group was 117 while those assigned to the SPAQ group was 118. There were 14 withdrawals or lost to follow up in the ASAQ group as opposed to 13 in the SPAQ group. Finally, 85 children completed the study in the ASAQ group and 80 in the SPAQ group. Follow-up was in accordance with the protocol.

The basic characteristics of the population as seen in Table 1, shows the different characteristics of the study participants on day 0 . All participants were positive for malaria after a thick blood smear $($ mean parasitaemia $=10799$ parasites $/ \mu$ l) on the first day of recruitment. The female-to-male ratio was 55:54 in the SPAQ group and 51:58 in the ASAQ group. Unfortunately, there were 8 unrecorded sex status in the SPAQ group and 9 in the ASAQ group. There was no significant difference in sex and age between both groups. The mean hemoglobin level at baseline was $10.24 \pm 2.3 \mathrm{~g} / \mathrm{dl}$ in the SPAQ group and $10.14 \pm 2.4 \mathrm{~g} / \mathrm{dl}$ in the ASAQ group. There was however no significant difference between the two groups $(\mathrm{p}=0.75)$. The mean temperature in the ASAQ group was $38.6 \pm 0.9^{\circ} \mathrm{C}$ and $37.9 \pm 0.8^{\circ} \mathrm{C}$ in the SPAQ group. This difference was also not significant between the two groups $(p=0.47)$. No significant difference was observed in the mean geometric levels of parasite counts in both treatment groups $(\mathrm{p}=0.38)$. In total, all the variables were not statistically significant in both treatment groups. Therefore, the two groups were statistically comparable at baseline (Table 1).

Table I Baseline Characteristics of the enrolled study participants

\begin{tabular}{|c|c|c|c|}
\hline \multicolumn{4}{|l|}{ Variable Treatment Group } \\
\hline & $\begin{array}{l}\text { Artesunate- } \\
\text { Amodiaquine (ASAQ) }\end{array}$ & $\begin{array}{l}\text { Sulfadoxine-Pyrimethamine- } \\
\text { Amodiaquine (SPAQ) }\end{array}$ & p-value \\
\hline Female: Male ratio* & $55: 54: 00$ & $51: 58: 00$ & 0.84 \\
\hline North: Far North & $54: 63$ & $55: 63$ & 0.94 \\
\hline Mean age (months) \pm SD & $48.01 \pm 28.7$ & $46.37 \pm 31.4$ & 0.69 \\
\hline Mean $\mathrm{Hb}(\mathrm{g} / \mathrm{dl}) \pm \mathrm{SD}$ & $10.24 \pm 2.3$ & $10.14 \pm 2.4$ & 0.75 \\
\hline Mean temperature $\left({ }^{\circ} \mathrm{C}\right) \pm \mathrm{SD}$ & $38.6 \pm 0.9$ & $37.9 \pm 0.8$ & 0.47 \\
\hline Weight(kg) $\pm S D$ & $|5.3| \pm 7.5$ & $|4.92 \pm 6|$. & 0.68 \\
\hline Geometric mean Parasite count $(/ \mu \mathrm{l}) \pm \mathrm{SD}$ & $3981 \pm 3$ & $380 I \pm 3.1$ & 0.38 \\
\hline
\end{tabular}

F:M , female: male ratio; * indicates that sex status missing for 8 in SPAQ group and 9 in ASAQ group; Hb, hemoglobin; SD, standard deviation. 


\section{Allelic, genotype and phenotype frequencies of the cyp2C8 gene}

We determined that there were 2 alleles in our study population with the predominance of the wild-type $\operatorname{cyp} 2 \mathrm{C} 8 * 1$ allele, at a frequency of $76 \%$ (Figure 1). In this study population of cyp $2 C 8$, we could distinguish between fast, intermediate and slow metabolisers with a predominance of fast metabolisers at a frequency of $70 \%$ (Figure 2).

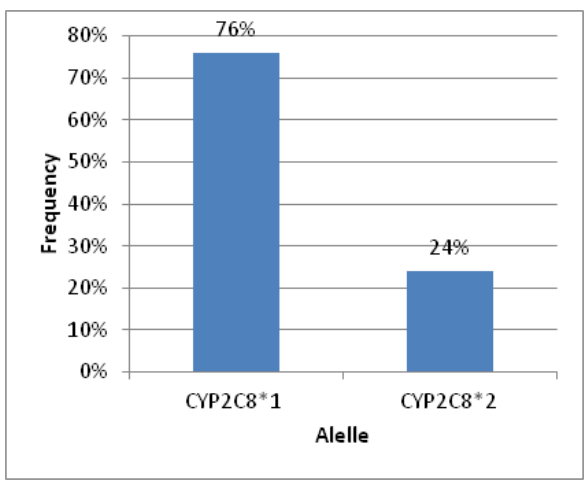

Figure I Distribution of alleles of cyp2C8.

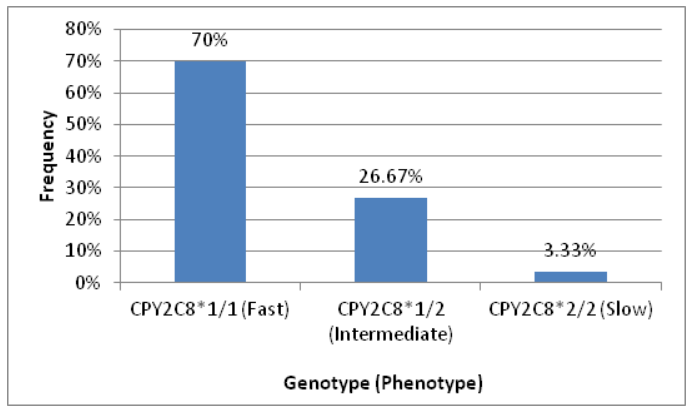

Figure 2 Frequency of сур2C8 genotype and phenotypes among study participants.

\section{Allelic, genotype and phenotype frequencies of the nat2 gene}

The nat $2 * 4$, nat $2 * 5$, nat $2 * 6$ and nat $2 * 7$ alleles were found, with nat $2 * 6$ being predominant at $39 \%$ and allele nat $2 * 7$ less found at $4 \%$ (Figure 3), of which nat $2 * 5 / 6$ was dominant at $28.12 \%$ (Figure 4 ). Slow metabolizers (nat $2 * 5 / 5$, nat $2 * 5 / 6$, nat $2 * 5 / 7$, nat $2 * 6 / 6$, nat $2 * 6 / 7$ ) were found at a frequency of $61.4 \%$ and intermediate metabolizers $($ nat $2 * 4 / 5$, nat $2 * 4 / 6$, nat $2 * 4 / 7)$ at $28.2 \%$ (Figure 5).

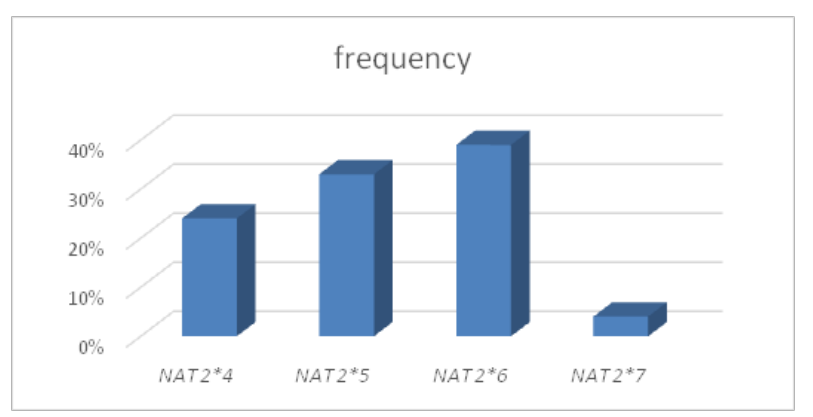

Figure 3 Allelic frequency of nat2 in Garoua.The nat2*4, nat2*5, nat2*6 and nat $2 * 7$ alleles were found, with nat2*6 being predominant $(39 \%)$ and allele nat $2 * 7$ less found $(4 \%)$.

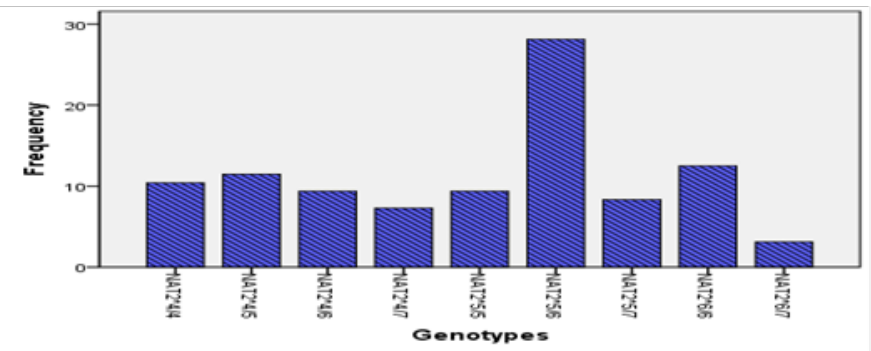

Figure 4 Genotypic frequencies of nat2 gene in Garoua.

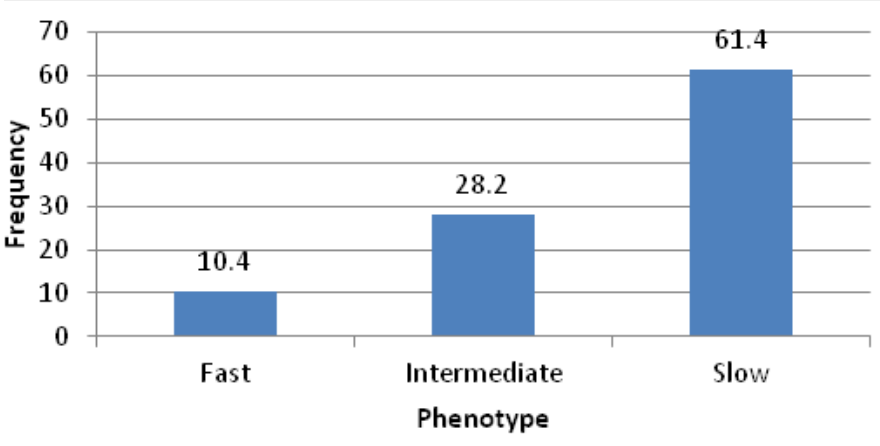

Figure 5 Phenotypic frequencies of nat2 gene in Garoua.

The genotypes nat $2 * 4 / 4$, nat $2 * 4 / 5$, nat $2 * 4 / 6$, nat $2 * 4 / 7$, nat $2 * 5 / 5$, nat $2 * 5 / 6$, nat $2 * 5 / 7$, nat $2 * 6 / 6$ and nat $2 * 6 / 7$ were found in Garoua among which nat $2 * 5 / 6$ was most dominant $(28.12 \%)$.

\section{Treatment outcomes}

The treatment outcomes were classified before PCR corrections, according to the WHO Guidelines as Adequate Clinical and Parasitological Response (ACPR), Early Treatment Failure (ETF), and Late Parasitological Failure (LPF). ACPR to SPAQ was much more dominant $(63.5 \%)$, with respect to ETF $(24 \%)$ and LPF $(12.5 \%)$. The ASAQ drug demonstrated an ACPR of $98 \%$ (Figure 6).

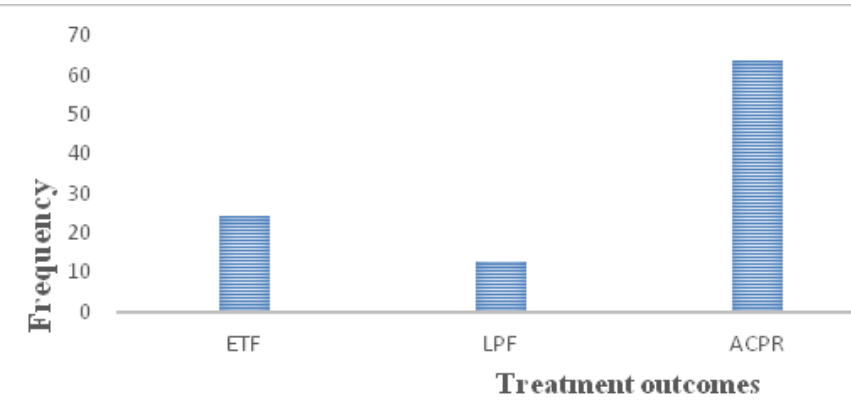

Figure 6 Frequency of various treatment outcomes SPAQ.

\section{Treatment outcome following ACTs administration and association with cyp2C8 and nat2 alleles}

ACPR was much more dominant $(63.5 \%)$, with respect to ETF (24\%) and LPF (12.5\%) (Table 2). Individuals with the fast acetylation were much more susceptible to experience early treatment failures (Odd's Ratio $=4.792$ and $p$-value $=0.020$ ) with respect to individuals with the slow and intermediate phenotype (Table 3) (Table 4). 
Table 2 Relationship between cyp2C8 phenotypes and treatment outcomes

\begin{tabular}{llllll}
\hline \multirow{2}{*}{ Phenotype } & \multirow{2}{*}{ No. examined (\%) } & \multicolumn{2}{l}{ Treatment outcomes (\%) } & Significance \\
\cline { 3 - 5 } & & ETF & LPF & ACPR & \\
\hline Slow & $3(3.3)$ & $0(0.0)$ & $3(100.0)$ & $0(0.0)$ & \\
Intermediate & $24(26.7)$ & $23(95.8)$ & $0(0.0)$ & $1(4.2)$ & $\mathrm{P}=0.000$ \\
Fast & $63(70.0)$ & $0(0.0)$ & $0(0.0)$ & $63(100.0)$ & \\
\hline
\end{tabular}

ETF, early treatment failure; LPF, late parasitological failure; ACPR, adequate clinical and parasitological response

Table 3 Relationship between nat2 phenotypes and treatment outcomes

\begin{tabular}{llllll}
\hline \multirow{2}{*}{$\begin{array}{llll}\text { Phenotype } \\
\text { No. examined (\%) }\end{array}$} & \multicolumn{2}{l}{ Treatment outcomes (\%) } & Significance \\
\cline { 3 - 5 } & & ETF & LPF & ACPR & \\
\hline Slow & $66(68.75)$ & $0(0.0)$ & $3(100.0)$ & $0(0.0)$ & \\
Intermediate & $21(21.88)$ & $23(95.8)$ & $0(0.0)$ & $1(4.2)$ & $\mathrm{P}=0.02$ \\
Fast & $9(9.37)$ & $0(0.0)$ & $0(0.0)$ & $63(100.0)$ & \\
\hline
\end{tabular}

ETF, early treatment failure; LPF, late parasitological failure; ACPR, adequate clinical and parasitological response

Table 4 Relationship between nat2 Phenotypes and LPF

\begin{tabular}{llllll}
\hline \multirow{2}{*}{ Phenotype } & \multicolumn{6}{l}{ LATE PARASITOLOGICAL FAILURE (LPF) } \\
\cline { 2 - 6 } & Present & Absent & OR & $\mathbf{9 5 \%} \mathbf{~ C I}$ & p-value \\
\hline Slow & 2 & 19 & 0.684 & $0.138-3.395$ & 0.641 \\
Fast & 6 & 3 & $4.333^{*}$ & $0.921-20.379$ & $0.047^{*}$ \\
Intermediate & 7 & 59 & 0.593 & $0.172-2.048$ & 0.405 \\
\hline
\end{tabular}

OR, Odd's ratio; $\mathrm{Cl}$, confidence interval; (*), statistically significant

\section{Clustering analysis}

The distance tree presents three clusters:

a. Cluster A - of patients who are intermediate metabolizers with wild type Pfdhfr-S108 and Pfdhps-C58, (without resistance markers), b. Cluster B - of patients who are slow metabolizers with the 2 resistance markers $108 \mathrm{~N} \& 58 \mathrm{R}$

c. Cluster $\mathrm{C}$ - of patients who are rapid metabolizers, without the resistance markers S108 \& C58

As depicted in Figure 7, slow metabolizers clustered with resistance mutation (cluster $\mathrm{B}$ )

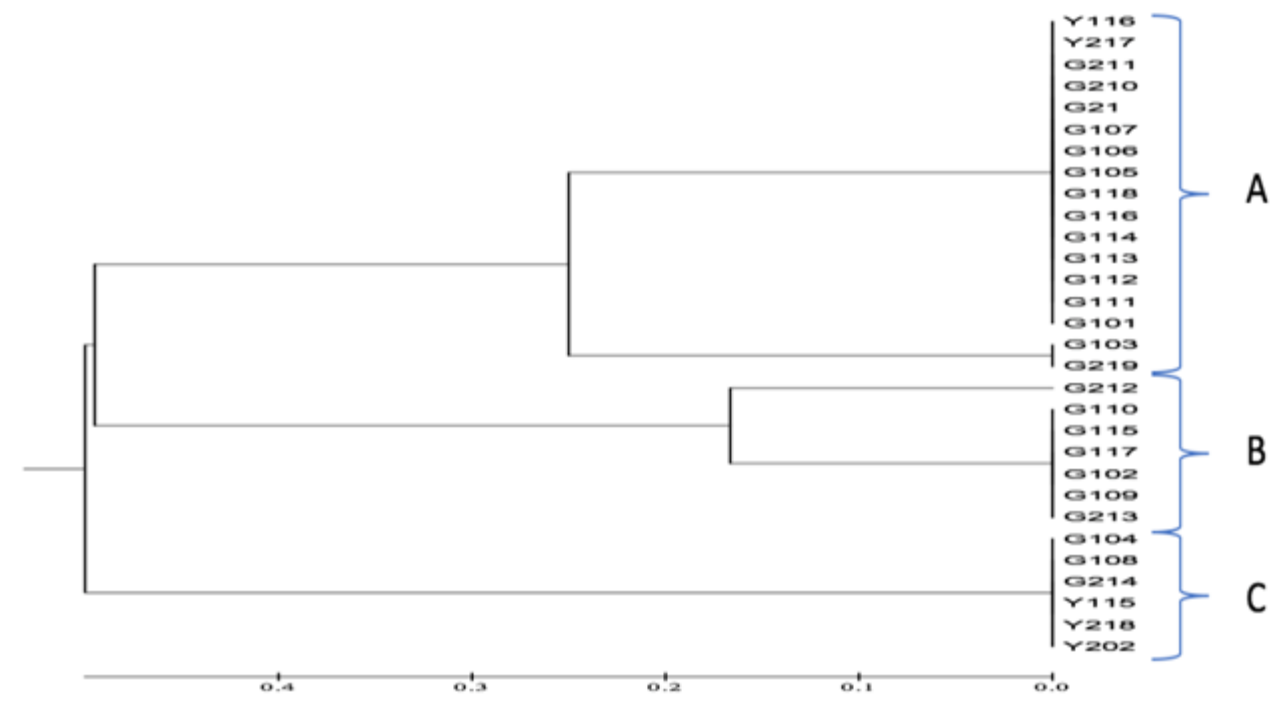

Figure 7 Distance tree of patient's phenotypic status according to nat 2, Pfdhfr and Pfdhps genes.

A, intermediate metabolizers without resistance mutations; B, slow metabolizers with resistance mutations; C, fast metabolizers without resistance mutations; Yagoua (Y) \& Garoua (G) sites. 


\section{Discussion}

Malaria remains a cause of mortality and morbidity worldwide. The consensus view of recent studies and reviews is that, malaria accounts for about 429,000 deaths annually, Sub-Saharan Africa bearing the heaviest burden. In Cameroon, malaria is also a public health problem, responsible for about $49 \%$ of hospitalisations. Artemisininbased combination treatments are now generally accepted as the best treatments for uncomplicated falciparum malaria since the early 2000 and implemented in Cameroon in 2006. They have a rapid and reliable efficacy, although several cases of poor treatment outcomes as well as adverse effects have been noted in some patients. One of the challenges in the effective treatment outcomes is mutant polymorphisms in genes encoding enzymes responsible for metabolism of ACTs, notably the cyp $2 \mathrm{C} 8$ and nat 2 gene, wherein individuals could either be slow, fast or intermediate metabolizers.

In our study population, $44.8 \%$ of patients were men and $55.1 \%$ were women. This could be explained by the natural predominance of women in any given population. Malaria was prevalent in both sexes with the mean parasitaemia being 10,799 parasites $/ \mu \mathrm{L}$. This study allowed for the characterization of cyp $2 \mathrm{C} 8$. The relatively low frequency of the cyp2C8*2 allele (24\%) observed in this study population is similar to that observed in Senegal (15\%) and Madagascar (15\%), ${ }^{15}$ Tanzania (19\%), ${ }^{16}$ Burkina Fasso $(15.5 \%)^{17}$ and Ghana $(17.5 \%) .{ }^{18}$ The genotypic frequencies observed in the study population are partially consistent with other available data: the cyp $2 C 8 * 1 / 1(70 \%)$ genotype obtained, corresponding to the rapid metabolisers, corroborates those observed in several countries, particularly in Senegal (55.7\%), Uganda (79.7\%), Madagascar $(73.7 \%)$ and $9 \%) .{ }^{15}$ The frequency of the cyp $2 C 8 * 1 / 2$ genotype $(26.67 \%)$ corresponding to the intermediate metaboliser phenotype is on the same line as that obtained in Senegal $(44.3 \%)^{15}$ but different from those observed in Uganda (19.9\%) and Madagascar (22.2\%). ${ }^{15}$

In this study, nat $2 * 4$, nat $2 * 5$, nat $2 * 6$ and nat $2 * 7$ were found in the north of Cameroon. nat $2 * 6$ was predominant $(39 \%)$ whereas nat $2 * 7$ was the least found (4\%), compared with findings in Bangolan, wherein $n a t 2 * 6$ was found at $24.2 \% .{ }^{19}$ The $n a t 2 * 6$ allele was among the most frequent alleles in populations of European and African descent, whereas $n a t 2 * 7$ was specific to the Asian population. ${ }^{20,21}$ With respect to nat 2 gene phenotypes, intermediate metabolizers were dominant (61.4\%) in the north of Cameroon. This results were similar to those obtained previously in Garoua, Mutengene and Bangolan, where slow and intermediate metabolizers dominated. ${ }^{22}$ Furthermore, studies in the north, east and west of Nigeria as well as in Kenya assessing the polymorphism of the nat 2 gene had similar results in accordance with findings showing that Africans are predominantly slow and intermediate metabolizers. ${ }^{16,23}$

An inter-individual difference in drug metabolism rate is likely due to human genetic polymorphisms. ${ }^{24}$ With this in mind, we sought to determine the relationship between metabolism rates and treatment outcomes. According to our study, individuals with a nat2 fast metabolizer status seem to experience early treatment failures $(\mathrm{OR}=4.792, \mathrm{p}=0.02)$, contrary to those with a slow or intermediate metabolizer status. This relationship indicates that rapid metabolizers are more likely to have poor treatment outcomes. With regard to late parasitological failures, again, individuals with the nat 2 fast metabolizer status were liable to have late parasitological failures $(\mathrm{OR}=4.333, \mathrm{p}=0.047)$, contrary to those with the slow and intermediate metabolizer status. This is in concordance with a 6-months trial conducted in Japan, on tuberculosis patients using Isoniazid, in which patients with the fast metabolizer status were more susceptible to early treatment failures. ${ }^{25}$ While individuals with a fast metabolizer status were prone to poor treatment outcomes (early treatment failures and late parasitological failures), those with a slow and intermediate metabolizer status were much more susceptible to experiencing adverse effects to the prescribed drugs as demonstrated by. ${ }^{22}$ This can be explained by the fact that fast acetylators metabolize xenobiotics faster than the required time for therapeutic action to occur, hence, early treatment failures or late parasitological failures. Slow and intermediate acetylators on the other hand metabolize and eliminate xenobiotics slower than normal which may expose the human being to drug toxicity from accumulation of metabolites, and leading to adverse effects. ${ }^{19,25}$ The clustering pattern of responders is indicative of the fact that slow metabolizers clustered with the resistance mutation genes; this is not the case for rapid and intermediates metabolizers and the reasons for this observation needs to be investigated further.

\section{Conclusion}

From the investigation of the influence of single nucleotide polymorphisms in the cypC28 and nat 2 genes on treatment outcomes in patients suffering from uncomplicated malaria in the north of Cameroon, we found that, there are two allelic frequencies with the wild type of cyp $2 C 8 * 1$ being at $76 \%$ and the cyp $2 C 8 * 2$ at $24 \%$. Of these $70 \%$ were fast metabolizers. No trend with respect to treatment response was found for the cyp2C8. However, the main genotype in the north was nat $2 * 5 / 6$ for the acetylators, with the slow metabolizer status being the main phenotype found at $61.4 \%$ which also clustered with adequate clinical and parasitological response to antimalarial drugs.

\section{Authors' contributions}

WFM, RNM, JPC, AN, IA, OA contributed to the design of the study. WFM coordinated the study. RNM, IA, supervised the enrolment, clinical coordination and follow-up of patients. AMN, JPC, EMA participated in data entry, collection and analysis of data and writing up the manuscript alongside RNM, JPC, DNM, performed the molecular analysis. All authors contributed in the revision of the manuscript and approved the final version of the manuscript prior to submission.

\section{Acknowledgments}

Thanks to Marc Yannick Mvondo, Data processer for the layout of the document. My research project was partially funded by the National Drug Quality Control and Valuation Laboratory (LANACOME), the National Program for Malaria Control Cameroon (PNLP), The Biotechnology Centre of the University of Yaounde I, The Gates Malaria Partnership, LSHTM, UK, with main funding through WFM by the Malaria Research Capacity Development for West and Central Africa (MARCAD) of the DELTAS Africa Initiative. The DELTAS Initiative is an independent funding scheme of the African Academy of Sciences (AAS)'s Alliance for Accelerating Excellence in Science in Africa (AESA) and supported by the New Partnership for Africa's Development Planning and Coordination Agency (NEPAD Agency) with funding from the WELLCOME Trust $[10774 / \mathrm{A} / 15 / \mathrm{Z}$ of March 30, 2016] and the UK government. The views expressed in this publication are those of the author(s) and not necessarily those of AAS, NEPAD Agency, Wellcome Trust or the UK government.

\section{Conflicts of interest}

Authors declare that there is no conflict of interest. 


\section{References}

1. Fact Sheet: World Malaria Report. WHO; 2015.

2. Songue E, Tagne C, Mbouyap P, et al. Epidemiology of Malaria in three Geo-Ecological Zones along the Chad-Cameroon Pipeline. Am J Epidemiol Infect Dis. 2013;1(4):27-33.

3. Maiga H, Djimde AA, Beavogui AH, et al. Efficacy of sulphadoxine-pyrimethamine + artesunate, sulphadoxine-pyrimethamine + amodiaquine, and sulphadoxine-pyrimethamine alone in uncomplicated falciparum malaria in Mali. Malaria Journal. 2015;14:64.

4. Mbacham WF, Evehe MS, Akaragwe IA, et al. Therapeutic Efficacy of Amodiaquine, Sulfadoxine-Pyrimethamine and the Combination Amodiaquine and Sulfadoxine-Pyrimethamine for the Treatment of Uncomplicated Falciparum Malaria in Children in Cameroonian. Malaria Journal. 2010;9:34-44.

5. Nambei WS, Achonduh O, Bogon A, et al. Genetic Diversity Of Field Isolates From Bangui, Central African Republic: Plasmodium falciparum. Rev CAMES-Série A. 2013;13(Suppl 2):77-81.

6. Tekete MM, Toure S, Fredericks A, et al. Effects of amodiaquin and artesunate on sulphadoxine-pyrimethamine pharmacokinetic parameters in children under five in Mali. Malar J. 2011;10:275.

7. Report of the technical consultation on seasonal malaria chemoprevention (SMC). WHO: Geneva; 2011. 11 p

8. Seasonal Malaria Chemoprevention with Sulfadoxine-Pyrimethamine plus Amodiaquin in Children: A Field Guide. WHO: Geneva; 2013. 56 p.

9. Teixeira RL de F, Morato RG, Cabello PH, et al. Genetic polymorphisms of NAT2, CYP2E1 and GST enzymes and the occurrence of antituberculosis drug-induced hepatitis in Brazilian TB patients. Mem Inst Oswaldo Cruz. 2011;106(6):716-724

10. Methods for surveillance of antimalarial drug efficacy. WHO: Geneva; 2009. 90 p.

11. Plowe CV, Djimde A, Bouare M, et al. Pyrimethamine and proguanil resistance-conferring mutations in Plasmodium falciparum dihydrofolate reductase: polymerase chain reaction methods for surveillance in Africa. Am J Trop Med Hyg. 1995;52(6):565-568.

12. Dai Z, Weichenhan D, Wu YZ, et al. An AscI boundary library for the studies of genetic and epigenetic alterations in $\mathrm{CpG}$ islands. Genome Res. 2002;12(10):1591-1598.

13. Chen M, Xia B, Chen B, et al. N-acetyltransferase 2 slow acetylator genotype associated with adverse effects of sulphasalazine in the treatment of inflammatory bowel disease. Can J Gastroenterol. 2007;21(3):155-158.

14. Yokogawa K, Nakaharu T, Ishizaki J, et al. Kinetic phenotypic diagnosis of $\mathrm{N}$-acetylation polymorphism in patients based on ratio of urinary metabolites of salicylazosulfapyridine. Int J Pharm. 2001;229(1-2):183191
15. Paganotti GM, Gramolelli S, Tabacchi F, et al. Distribution of human CYP2C $8 * 2$ allele in three different African populations. Malaria Journal. 2012;11(1):125.

16. Marwa KJ, Schmidt T, Sjögren M, et al. Cytochrome P450 single nucleotide polymorphisms in an indigenous Tanzanian population: a concern about the metabolism of artemisinin-based combinations. Malaria Journal. 2014;13:420.

17. Parikh S, Ouedraogo JB, Goldstein JA, et al. Amodiaquine Metabolism is Impaired by Common Polymorphisms in CYP2C8: Implications for Malaria Treatment in Africa. Clinical Pharmacology \& Therapeutics. 2007;82(2):197-203.

18. Kudzi W, Dodoo AN, Mills JJ. Characterisation of CYP2C8, CYP2C9 and CYP2C19 polymorphisms in a Ghanaian population. BMC Med Genet. 2009; 10:124

19. Achonduh OA, Atogho-Tiedeu B, Mbulli IA, et al. Adverse Events Clustering with NAT2 Slow Metabolisers following Deparasitization in Children in Bangolan, NWR Cameroon. Journal of Life Sciences. 2013;7(7):742-748.

20. Mahasneh A, Jubaili A, El Bateiha A, et al. Polymorphisms of arylamine $\mathrm{N}$-acetyltransferase 2 and risk of lung and colorectal cancer. Genet Mol Biol. 2012;35(4):725-733.

21. Hein DW. N-acetyltransferase 2 genetic polymorphism: Effects of carcinogen and haplotype on urinary bladder cancer risk. Oncogene. 2006;25(11):1649-1658.

22. Kengne JPC, M Nji Akindeh, M Ali Innocent, et al. Predominance of $\mathrm{N}$-acetyl transferase 2 slow acetylator alleles in children less than ten years experiencing adverse treatment events following artemisinin-based combination therapy in North and South West Regions of Cameroon. African Journal of Biotechnology. 2016;15(25):1285-1291.

23. Lin HJ, Han CY, Lin BK, et al. Ethnic distribution of slow acetylator mutations in the polymorphic N-acetyltransferase (NAT2) gene. Pharmacogenetics. 1994;4(3):125-134.

24. Belle DJ, Singh H. Genetic Factors in Drug Metabolism. Am Fam Physician. 2008;77(11):1553-1560.

25. Azuma J, Ohno M, Kubota R, et al. NAT2 genotype guided regimen reduces isoniazid-induced liver injury and early treatment failure in the 6-month four-drug standard treatment of tuberculosis: A randomized controlled trial for pharmacogenetics-based therapy. Eur J Clin Pharmacol. 2013;69(5):1091-1101. 